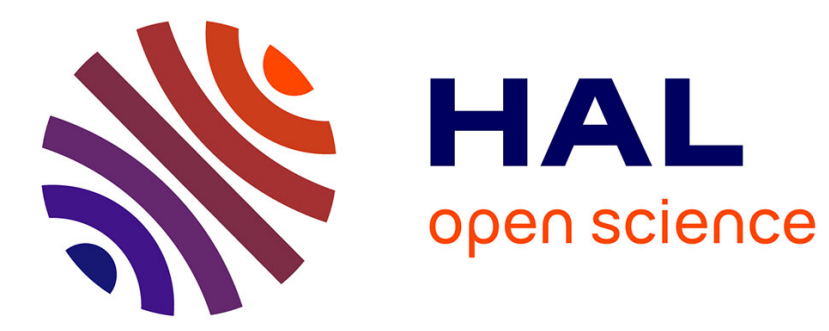

\title{
Les voies de la réforme du secteur gazier russe
}

Catherine Locatelli

\section{To cite this version:}

Catherine Locatelli. Les voies de la réforme du secteur gazier russe. Revue d'Etudes Comparatives Est-Ouest, 2013, 44 (1), pp.153-167. 10.4074/S033805991300106X . halshs-00822857

\section{HAL Id: halshs-00822857 https://shs.hal.science/halshs-00822857}

Submitted on 15 May 2013

HAL is a multi-disciplinary open access archive for the deposit and dissemination of scientific research documents, whether they are published or not. The documents may come from teaching and research institutions in France or abroad, or from public or private research centers.
L'archive ouverte pluridisciplinaire HAL, est destinée au dépôt et à la diffusion de documents scientifiques de niveau recherche, publiés ou non, émanant des établissements d'enseignement et de recherche français ou étrangers, des laboratoires publics ou privés. 


\title{
ÉCONOMIE DU DÉVELOPPEMENT DURABLE ET DE L'ÉNERGIE
}

\section{Les voies de réforme du secteur gazier russe}

\author{
Catherine Locatelli
}

juin 2012

Cahier de recherche $n^{\circ} 9 / 2012$ 



\title{
Les voies de réforme du secteur gazier russe
}

\author{
C. L ocatelli ${ }^{1}$, juin 2012
}

\section{Résumé:}

Le secteur gazier russe est aujourd'hui l'objet d'évolutions en profondeur même s'il reste dominé par la puissante société Gazprom, détenue par l'Etat. Ces évolutions se caractérisent principalement par une restructuration de $\mathrm{G}$ azprom, des hausses conséquentes de prix et surtout I'introduction de formes concurrentielles qui tendent à normer le comportement de Gazprom.

Cette réorganisation ne suit cependant pas le modèle de restructuration des industries gazières mises en œuvre dans I'UE. Ce modèle est principalement basé sur la dé-intégration des monopoles verticalement intégrés et des marchés concurrentiels. Alors que c'était le projet initial (discuté en 2002), ce modèle s'avère de fait dans les conditions institutionnelles et économiques de la Russie impossible à mettre en œuvre.

\section{Abstract :}

The Russian gas sector is characterised by some important evolution even if the State Gazprom Company remains the main gas producer in Russia. These evolutions are principally: a restructuration of Gazprom; big increases in Russian gas prices; introduction of some competition to try to normalize the Gazprom's behaviours.

This reform doesn't follow the EU gas restructuration model. This model is based on the unbundling of the vertically gas companies and competitive markets. The initial project (discussed in 2002) of the Russian gas reform was to implement the EU gas model. But in the Russian institutional and economic environment this type of reform seems impossible to implement.

Le marché gazier russe est depuis le début des années 1990 dominé par la holding Gazprom née de la restructuration du ministère du gaz de l'U nion soviétique. Les contraintes auxquelles se heurte cette société dans ses stratégies d'adaptation face aux évolutions de son principal marché d'exportation, celui de I'U nion européenne, mettent au premier plan la question de la réforme de Gazprom et plus largement de l'industrie gazière russe. Le modèle de plus en plus concurrentiel du marché gazier européen place au centre du débat la question de l'efficience et de la flexibilité des stratégies d'exportation de Gazprom, en particulier au niveau de ses relations contractuelles avec ses partenaires européens ${ }^{2}$.

\footnotetext{
${ }^{1}$ Chercheur CNRS, EDDEN, CNRS-U niversité de Grenoble, CNRS, BP 47, F-38040 Grenoble cedex 9 E mail : catherine.locatelli@ upmf-grenoble.fr

Tel : $334 \overline{456528575}$

${ }^{2}$ La question de la modification de la durée des contrats de long terme et de l'introduction d'un prix spot dans la formule d'indexation des prix de ces contrats est ainsi au centre des débats.
} 
Cette question n'est pas nouvelle. Commencée sous M. Gorbatchev et poursuivie en 1991 et 1992, la restructuration de ce secteur fait l'objet d' un débat récurrent qui oppose les tenants de la centralisation, en faveur du maintien de Gazprom (à condition que celui-ci soit quelque peu réformé), à ceux de la libéralisation de l'industrie. Pour ces derniers, il s'agissait principalement de mettre en œuvre le modèle dé-intégré et concurrentiel des industries gazières utilisé dans I'UE au travers de ses deux directives gazières et du " $3^{\mathrm{e}}$ paquet Energie ». La faisabilité et la crédibilité d'une réforme calquée sur celle des industries gazières de l'UE a été au cœur des débats. La réforme des structures organisationnelles est ainsi intrinsèquement liée à celle, de grande ampleur, des prix du gaz sur le marché intérieur russe.

II apparaît clairement que l'environnement économique et institutionnel de la Russie ne permet guère encore à ce jour d'envisager la mise en place $d$ ' un modèle qui impliquerait une dé-intégration (unbundling) totale de Gazprom. II n'en demeure pas moins que progressivement se dessinent les contours d'un marché gazier russe à l'avenir sensiblement plus concurrentiel, ouvrant ainsi la voie à une réforme en profondeur de ce secteur. Depuis le début des années 2000, le marché gazier russe connait des évolutions en profondeur qui modifient durablement la structure de cette industrie et tendent à opérationnaliser, au moins dans certains de ses grands principes (prix et concurrence), les projets de réforme de 20002002. L'objectif de cet article est double. II est d'abord d'analyser les spécificités économiques et institutionnelles de la Russie qui empêchent la mise en œuvre d'un modèle dé-intégré et concurrentiel d'organisation de son industrie gazière. II est ensuite de caractériser ce marché et de présenter les principales voies qu'emprunte aujourd'hui sa réforme.

\section{Un secteur dominé par l'entreprise verticalement intégrée G azprom.}

A l'inverse des principes concurrentiels et de privatisation mis en œuvre dans le secteur pétrolier, les réorganisations de 1991 et 1992 ont fait émerger dans le secteur gazier une entreprise dominante ayant un pouvoir de marché considérable (Tarr, 2010), Gazprom. holding constituée sur la base de l'ancien ministère du gaz soviétique, détient les actions de neuf sociétés de production, et les actions de la société de transport (Transgaz) et d'exportation, GazExport. Elle est à ce titre une entreprise verticalement intégrée de la production jusqu'à la distribution en passant par le transport et l'exportation (L ocatelli, 2003). En 1993, elle assure 93 \% de la production gazière russe et détient le monopole du transport par gazoduc et des exportations.

L'Etat est alors, avec $38 \%$, un actionnaire dominant mais non majoritaire de l'entreprise. II ne le deviendra qu' au début des années 2000 avec $51 \%$ des actions de la compagnie. A côté de Gazprom subsiste un certain nombre de compagnies gazières «indépendantes » et les compagnies pétrolières russes détentrices de réserves gazières, leur part en termes de production étant cependant marginale $(7 \%)$, même si elles détiennent des réserves significatives.

Toutefois dès le milieu des années 1990, suite notamment à la stagnation de la production gazière, différents arguments sont avancés pour justifier une réforme de l'entreprise dominante, et plus généralement de l'ensemble du secteur gazier. Le principal d'entre eux a trait à l'inefficience supposée de Gazprom. Celle-ci s'expliquerait de part son caractère de quasi-monopole et son statut d'entreprise ayant un actionnariat dominé par l'E tat. Un certain nombre de courants théoriques et quelques rares travaux relatifs aux performances de 
Gazprom sont mobilisés pour le démontrer. La théorie des droits de propriété et son prolongement dans la théorie de l'agence, la théorie du Public Choice et celle de la régulation (Wolf, 2009) justifient la meilleure performance des entreprises privées par rapport aux entreprises publiques. Des droits de propriété privés sont ainsi censés définir un système d'incitations susceptible d'orienter les comportements des agents vers la recherche de I'efficacité. Par ailleurs, les études empiriques relatives à la compagnie gazière mettent en exergue trois principales caractéristiques sur lesquelles vont s'appuyer les tenants de I'inefficience de Gazprom, à savoir les problèmes de Corporate Governance (problèmes d'agence), le « retournement » de I'agent contre son actionnaire principal, Gazprom étant souvent qualifié d'Etat dans l'Etat (Nemtsov \& M ilov, 2008) et ses mauvaises performances économiques.

A priori, la structure du capital de Gazprom permet de conclure que les problèmes de Corporate Governance liés à un actionnariat fractionné et dominé par les insiders sont moins prégnants que dans les schémas de privatisation initiés dans le secteur pétrolier russe (Earle, Estrin, 1996). Toutefois, les relations entre la holding et ses sociétés de production ou de transport ont été particulièrement opaques (A hrend \&Tompson, 2004 ; Stern, 2005). Deux mécanismes se sont ainsi substitués aux relations de propriété, renforçant la centralisation de la prise de décision (notamment en matière d'allocation des ressources) au niveau de la seule holding et non plus au niveau de ses sociétés. II s'agit d'une part des prix de cession interne qui contribuent à maintenir les sociétés de production en déficit (K ryukov \& Moe, 1996). D'autre part, le fonds de stabilisation et d'investissements établi au niveau de la holding et en partie financé par les revenus à l'exportation permet d'orienter les stratégies d'investissement des sociétés de production au travers de sa capacité de financement (Sorokine, Eskin \&Wilson, 1995).

Ces études soulignent également les mauvaises performances économiques de la compagnie, avec notamment un retour sur actif moindre que pour les compagnies pétrolières privées russes, un problème de suremploi ${ }^{3}$ et des coûts de production élevés. En particulier, elles mettent en exergue une détérioration de la situation financière de la compagnie dans les années 2000, avec une croissance de plus de $60 \%$ de son endettement entre 2005 et 2010. Ses dettes par rapport aux actifs augmentent sensiblement (W ood Mackenzie, 2004 ; Victor, 2008 ; Victor \& Sayfer, 2009). Cependant, depuis 2008, le profit net de la compagnie a eu tendance à croître, notamment sous l'effet de la hausse des prix du gaz sur le marché russe depuis le début des années 2000. II est en effet difficile d'évaluer la rentabilité de Gazprom, tant l'environnement institutionnel russe a induit un certain nombre de contraintes spécifiques. Les mauvaises performances financières de la holding résultent pour une large part d'une double logique, celle des non-paiements des années $1990^{4}$ et celle des bas prix réels du gaz.

\section{Les limites à la mise en cuvre du modèle concurrentiel et dé-intégré de l'UE en R ussie}

\footnotetext{
${ }^{3}$ L'étude de B. Nemtsov et V. M ilov (2008) met ainsi en exergue la hausse du nombre d'employés de Gazprom. En particulier sur la période 2003-2007, ceux-ci sont passés de 391000 à 445000.

${ }^{4}$ En 1996, seuls $72 \%$ des ventes de gaz ont été payées, chiffre qui n'est plus que de $45 \%$ en 1997 dont $6 \%$ seulement réglées en liquide. En 1996, 51 \% ont été réglées sous forme troc de en, chiffre de 39\% en 1997 (Bagratian \& Gürgen 1997 ; Stern 1998).
} 
Les projets de restructuration de l'industrie gazière russe de la fin des années 1990 et du début des années 2000, portés par un certain nombre de réformateurs, dont le ministre du développement économique et du commerce G. Greff, visaient dans leurs grandes lignes à mettre en place une réforme calquée sur celle des industries gazières de I'UE. S'inscrivant dans cette logique, ils étaient principalement centrés sur la dé-intégration verticale de la compagnie Gazprom ${ }^{5}$. II s'agissait de séparer les fonctions de production et de transport (selon une logique d'unbundling) afin d'isoler les segments concurrentiels de ceux en monopole naturel. Dans une perspective concurrentielle, il s'agissait égal ement de créer six producteurs à partir des sociétés de production détenues à $100 \%$ par Gazprom. Ce dernier réduit à sa seule fonction de transporteur, en monopole naturel régulé, détenu par l'Etat avec un accès des tiers au réseau devait par ailleurs garder sa fonction d' unique exportateur de gaz, seule concession par rapport au modèle européen. Dans une dernière étape (2007-2013), Gazprom aurait été cantonné aux seules activités d'exportation. La position des « réformateurs » était en effet beaucoup plus nuancée sur la question de la libéralisation des exportations gazières (notamment à destination de l'Europe), celle-ci ne faisant pas partie de leur priorité.

La faisabilité et la crédibilité d'une réforme basée sur le modèle dé-intégré et concurrentiel de I'UE ont toutefois posé question au regard des spécificités de l'environnement économique et institutionnel russe. Suivant les approches néo-institutionnalistes développées par North (1991) et Williamson (1996), on peut en effet émettre l'hypothèse que les structures de gouvernance et donc les modèles organisationnels sont dépendants des environnements institutionnels dans lesquels ils sont susceptibles d'être mis en œuvre. De ce point de vue, l'ineffectivité des droits de propriété privés en Russie, tout au moins jusqu'au début des années 2000 , met en cause la faisabilité et donc la crédibilité d' une telle réforme. La question de la sécurisation des droits de propriété privés en Russie a fait l'objet d'un vaste débat (Tompson, 2008; Runov, 2004; Hedlund, 2001), trois types d'incertitudes pouvant être identifiées. La première porte sur le droit d'usufruit sur les ressources. L'absence de libéralisation des prix énergétiques et le phénomène de non-paiement et de troc contraignent fondamentalement les droits de propriété sur les ressources, ainsi que les possibilités de retirer les recettes nécessaires de la vente sur le marché interne pour reconstituer les réserves et renouveler les infrastructures. Ces éléments définissent une situation de risque de « hold-up » caractérisée par O. Williamson (1985). Les opérateurs qui investissent sont totalement soumis au risque discrétionnaire des politiques de prix et à l'opportunisme d'une partie des acheteurs de gaz. La deuxième incertitude est relative aux droits de propriété sur les actifs des entreprises en raison de la faible légitimité du processus de privatisation (D eacon \& M ueller, 2004) et du fait de la manipulation de la loi sur les faillites. L'impossibilité de mettre en œuvre le mécanisme de faillite, en particulier en raison des non-paiements et des relations non monétaires, conduit à procéder aux transferts d'actifs à grande échelle indépendamment de la rentabilité des firmes, lorsqu'il est nécessaire de procéder à des restructurations (Locatelli \& Finon, 2003). Dernier élément, les droits de propriété privés sont mis en cause par le problème spécifique de la sécurisation des droits d'accès aux ressources naturelles. En dépit de la loi pétrolière (Subsoil Law) de 1992 et de ses amendements de 1995, le processus $d^{\prime}$ accès aux ressources en hydrocarbures est resté largement opaque (A dachi, 2009) ${ }^{6}$, objet de

\footnotetext{
${ }^{5}$ II s' agit notamment du projet élaboré en 2000 par le ministère du développement économique et du commerce, la commission fédérale de l'E nergie. En 2003, un autre projet du même type a été élaboré.

${ }^{6} 40 \%$ des licences allouées entre 1992 et 1995 I'ont été sans réelles obligations pour les utilisateurs (A dachi, 2009).
} 
réallocations discrétionnaires de la part de l'Etat des licences octroyées lors de la constitution des compagnies pétrolières et gazières.

La faisabilité et la crédibilité de la réforme sont également mises en cause par le système des prix gaziers russes qui empêche toute forme de concurrence. La politique de l'Etat concernant la structure des prix est d'abord contestée. Cette dernière intègre d'importantes subventions croisées entre le secteur industriel, et le secteur résidentiel, ce dernier étant subventionné par le premier. Ensuite, les bas prix internes du gaz naturel ${ }^{7}$ freinent la rentabilité de l'entreprise gazière. On peut estimer qu'ils ont été sur certaines périodes en moyenne inférieurs au coût marginal de production de Gazprom (Tarr \& Thomson, 2003 ; Dubek, Golub \& Strukova, 2006). Ces prix ne sont pas des prix de monopole et répondent à une logique de redistribution entre les consommateurs, ainsi qu' à la politique industrielle qu' entend mener I'E tat russe. Une autre forme de subventions a résidé jusqu' en 2000 dans le développement sur une échelle considérable des relations non monétaires: troc, non-paiement, crédit interentreprises. Dans ces conditions, les régulations du secteur gazier russe demeurent essentiellement de type quantitatif, en dehors de critères de rentabilité, de coût ou de prix. Elles s'apparentent à des mécanismes de rationnement. A insi, le marché est principalement géré par la négociation de quotas de consommation entre Gazprom et les grandes catégories de consommateurs de gaz (A hrend \& Tompson, 2004).

\section{L es voies de la réforme}

Les logiques économiques et organisationnelles du modèle Gazprom tendent aujourd'hui à être remises en question, notamment par les évolutions institutionnelles de son principal marché d'exportation, I'UE. La réforme de I'industrie gazière ne pouvant suivre la voie prônée par l'U nion européenne visant à la dé-intégration totale de Gazprom, elle emprunte d'autres logiques plus crédibles (et sans doute plus légitimes). Elle est principalement centrée sur une réforme des structures internes de Gazprom et sur l'émergence d'un certain degré de concurrence sur le marché russe. Celle-ci est une des voies permettant de normer le comportement de Gazprom. A l'inverse, il semble peu envisageable que sur le marché de I'UE Gazprom puisse être mis en concurrence avec d'autres acteurs russes. A fin de ne pas contribuer à la baisse des prix sur les marchés spot européens et de ne pas éroder le profit de monopole de la Russie sur ses exportations gazières, Gazprom pourrait maintenir son monopole d'exportation (Tarr, 2010).

\section{- La réorganisation de la compagnie gazière verticalement intégrée}

La première évolution notable de Gazprom a porté sur I'unification et la libéralisation de son marché d'actions en 2005, mouvement qui a accompagné la remontée de l'Etat jusqu'à $51 \%$. de son actionnariat. Elle s'est accompagnée d'une réorganisation interne progressive selon deux voies principales. La première vise à séparer, au sein de chaque filiale de Gazprom, les unités de production de celles de transport et de stockage. La deuxième voie de réorganisation a pour objectif de séparer les activités de base de la compagnie de celles qui sont annexes. Celles-ci doivent être regroupées dans des compagnies séparées (V ictor, 2008). La séparation des activités de Gazprom en différentes compagnies est la condition de la mise en œuvre d'un accès au tiers (ATR) prévu par la loi et d'une plus grande transparence des coûts de transports. Le réseau de Gazoduc étant, rappelons-le, un monopole naturel, I'ATR est le

\footnotetext{
${ }^{7}$ En 2001, ces prix étaient de $10 \$ / 1000 \mathrm{~m}^{3}$ pour les ménages et de 15 à $16 \$ / 1000 \mathrm{~m}^{3}$ pour les industriels, contre une moyenne de $120 \$ / 1000 \mathrm{~m}^{3}$ pour les exportations vers l'E urope de l'O uest.
} 
moyen de créer la concurrence dans le secteur gazier en organisant un accès libre et égalitaire au réseau de transport pour tous les producteurs. On doit par ailleurs noter l'existence d'un réel effort de transparence dans le domaine financier de la part de la compagnie gazière, lequel se traduit notamment par la publication régulière de comptes standards internationaux.

- La logique des doubles marchés : I'introduction d'une concurrence progressive

$M$ ais c'est principalement par l'introduction de certaines formes concurrentielles (et non par une réforme des droits de propriété) que I'Etat entend pour I'heure contraindre le comportement de Gazprom. La concurrence est, en effet, un moyen permettant de diminuer les asymétries d'information entre l'Etat et la compagnie gazière, et de faire révéler de manière indirecte de l'information à Gazprom. Deux grandes catégories de producteurs gaziers ont émergé à côté de Gazprom. II s'agit d' une part des compagnies pétrolières russes et d'autre part des "indépendants», compagnies privées détentrices de réserves gazières. Novatek en est l'une des plus importantes. Ces deux catégories, avec une production en hausse constante depuis 2005, ont représenté près de $22 \%$ de la production gazière russe en 2010 (cf. tableau 1). Gazprom ne détient donc plus le monopole de production de gaz en Russie.

Tableau 1 : R épartition de la production gazière russe entre les acteurs

\begin{tabular}{|l|l|l|l|l|l|l|l|}
\hline $\mathrm{G} \mathrm{m}^{3}$ & $\mathbf{2 0 0 5}$ & $\mathbf{2 0 0 6}$ & $\mathbf{2 0 0 7}$ & $\mathbf{2 0 0 8}$ & $\mathbf{2 0 0 9}$ & $\mathbf{2 0 1 0}$ & $\mathbf{2 0 1 1}$ (provisoire) \\
\hline Gazprom & 548,4 & 556,0 & 548,6 & 549,7 & 462,2 & 506,6 & 509,8 \\
\hline Autres producteurs & 92,2 & 100,2 & 104,1 & 113,9 & 120,2 & 141,7 & 160,7 \\
\hline Total & 640,6 & 656,2 & 652,7 & 663,6 & 582,4 & 650,3 & 670,5 \\
\hline
\end{tabular}

Source : Gazprom, ministère de l'énergie russe

L'émergence progressive de nouveaux acteurs gaziers s' est effectuée dans le cadre particulier de la mise en place d'un double marché, celui des prix régulés et celui des prix libres. L'approvisionnement gazier des ménages est assuré par le marché règlementé à des prix régulés par le «Federal Tariff Service ${ }^{8}$. Ce dernier définit selon des zones de prix définies géographiquement des tarifs de gros. Gazprom est le seul fournisseur de ce marché. Le secteur industriel (et en particulier le secteur électrique) peut quant à lui se fournir sur ce marché en fonction des quotas négociés avec Gazprom. A u-delà des quantités négociées, les consommateurs peuvent se fournir sur un marché qualifié de libre à des prix non régulés. $\mathrm{Ce}$ marché est approvisionné essentiellement par les indépendants gaziers, les compagnies pétrolières russes et à la marge par Gazprom, notamment pour son «nouveau gaz » (cf. encadré 1).

\section{Encadré 1 : Le marché gazier non régulé}

Le marché gazier non régulé est constitué :

- des ventes des indépendants et des compagnies pétrolière russes à des prix librement négociés. En 2007, les prix libres étaient 10 à $20 \%$ plus élevés que les prix négociés. Cette marge tend progressivement à disparaître.

\footnotetext{
${ }^{8}$ Quelques principales dispositions permettant à l'Etat de réguler les prix du gaz : la loi de 1995 sur les « monopoles naturels»

la loi de 1999 « sur l'approvisionnement gazier dans la Fédération de Russie » la directive de 1995 sur les prix du gaz, amendée en 1997, 2000 et 2010.
} 
- des ventes de Gazprom selon le schéma 5 + 5 sur la bourse d'échange gazière de la R ussie (marché spot) créée par le décret de 2006. Gazprom, au travers de sa filiale M ezhregiongaz, peut vendre à des prix libre sur cette plateforme d'échange de gaz $\left(5 \mathrm{Gm}^{3}\right)$ à condition qu'en contrepartie la même quantité de gaz soit vendue par les indépendants $\left(5 \mathrm{Gm}^{3}\right)$. La rationalité sous-jacente est que les ventes de Gazprom ne doivent pas dépasser les ventes des parties tiers. 2006-2007 et 2008 ont vu les échanges sur cette bourse croître de manière significative, fournissant des informations de plus en plus crédibles sur les prix de marché. La crise de fin 2008 a toutefois fortement freiné son développement. Demeure toutefois la volonté de la part des autorités publiques de créer une plateforme d'échange plus permanente.

- 2007 : décret permettant que le «nouveau gaz produit» (soit les nouveaux gisements mis en production), notamment par Gazprom, soit vendu à des prix libres. II résulte implicitement de cette règle que le volume de gaz que Gazprom vend à des prix régulés n' excède pas $300 \mathrm{G} \mathrm{m}^{3}$. A u-delà, les consommateurs doivent s'approvisionner à des prix libres auprès de Gazprom, des indépendants ou des compagnies pétrolières russes (Henderson, 2012).

A u total, Gazprom représenterait $25 \%$ des ventes effectuées sur le marché libre (soit plus de $100 \mathrm{Gm}^{3}$ ).

Ce mouvement de libéralisation partielle des prix au travers de la croissance des volumes échangés sur le marché non régulé s'accompagne d'une augmentation progressive des prix régulés dont l'enjeu principal est d'assurer la rentabilité de G azprom sur son marché intérieur. Cette augmentation s'inscrit dans un projet de réforme globale. Initialement, I'objectif poursuivi était que les prix intérieurs russes soient à parité (déduction faite des accises, taxes d'exportation et coût de transport) avec les prix européens à partir de 2011 pour le secteur industriel, et à partir de 2013 pour le secteur résidentiel. Une telle logique d'alignement sur les prix européens aurait induit des augmentations considérables, compte tenu de l'indexation des prix du gaz sur ceux du pétrole dans les formules de prix des contrats de long terme. D' une part, la crise financière et économique de 2008 a conduit à retarder ces objectifs, même si les prix régulés ont continué à augmenter. $D$ 'autre part, de telles hausses ne se justifient pas forcément d'un point de vue économique. En l'absence d'un marché mondial du gaz naturel et compte tenu de la position dominante de Gazprom sur le marché intérieur russe, le prix intérieur doit être celui qui permet aux producteurs de couvrir leur coût marginal de long terme (Tarr \& Thomson, 2003). Dans ces conditions, les prix sur le marché européen ne peuvent servir de benchmarking à la réforme des prix intérieurs. Cette parité ne doit pas être considérée comme un objectif réel, mais plutôt comme l'indication d' un engagement de l'Etat à augmenter les prix en interne (Henderson, 2011). II n'y a ainsi aucune raison économique à unifier les prix intérieurs russes avec ceux du marché européen ${ }^{9}$.

Tableau 2 : M oyenne des prix régulés du gaz naturel en R ussie2006-2011 Prévisions pour 2012 et 2013

\begin{tabular}{|c|c|c|c|c|c|c|c|c|c|}
\hline Par mille $\mathrm{m}^{3}$ & & 2006 & 2007 & 2008 & 2009 & 2010 & 2011 & $\begin{array}{c}2012 \\
(2)\end{array}$ & $2013(2)$ \\
\hline Industries & Roubles & 1104 & 1352 & 1690 & 1957 & 2478 & $\begin{array}{c}2583 \\
(1)\end{array}$ & - & - \\
\hline & Dollars & 40.58 & 52.81 & 67.87 & 64.80 & 82.60 & $91(2)$ & 105 & 119 \\
\hline
\end{tabular}

\footnotetext{
${ }^{9}$ Dans le cas où la R ussie chercherait un « prix unifié » en alignant le prix à l'export sur le coût marginal de long terme de Gazprom, elle enregistrerait des pertes financières de l'ordre de 5 à 7,5 milliards de $\$$ au profit du consommateur européen (Tarr \&Thomson, 2003). A l'inverse si en netback, elle prenait comme prix de référence le prix du marché européen, les augmentations qui en résulteraient conduiraient à des inefficiences importantes, le prix étant largement supérieur au coût marginal de long terme de Gazprom, d'où un profit de monopole important pour la compagnie gazière (Tarr, 2010).
} 


\begin{tabular}{|l|l|c|c|c|c|c|c|c|c|}
\hline M énages & Roubles & 863 & 1031 & 1291 & 1294 & 1903 & & & \\
\hline & Dollars & 31.72 & 40.27 & 51.85 & 49.47 & 63.43 & & & \\
\hline
\end{tabular}

(1) : Pour les six premiers mois de 2011

(2) : Prévisions du Federal Tariff Service de la Russie

Sources: Price rises and market reform in Russia, a long and winding road. G as matters, J une 2011 ; G azprom Financial report 2011, Gazprom 2012 ; Dynamics of gas wholesale prices for industrial consumers. Russian Federal Tariff Service, [consulté le 13.04.2012].

Deux facteurs permettent aujourd'hui de conclure que les prix actuels sont ceux qui permettent à Gazprom de commencer à couvrir son coût marginal de long terme, même si cela s'avère être un exercice difficile. Premièrement, la compagnie gazière est en état depuis 2009 de dégager un certain niveau de rentabilité en interne, à l'inverse de la situation des années 1990 et 2000. A insi, la hausse de son profit en 2010 est clairement liée à la croissance de ses revenus sur son marché intérieur, alors que ses revenus sur le marché européen ont eu tendance à stagner entre 2009 et 2010, et à nettement décroître par rapport à l' année record de 2008. Deuxièmement, certains producteurs (tel Novatek), même à des prix dérégulés, sont en état de concurrencer Gazprom sur certains segments de son marché (sur le segment électrique par exemple). Dès lors, on peut faire l'hypothèse que les niveaux des prix dérégulés ne sont guère éloignés de ceux des prix régulés et permettent de dégager un niveau de rentabilité suffisant (Henderson, 2010 et 2011). Cette évolution permet progressivement I'émergence d'un marché gazier russe plus concurrentiel, qui n'est plus géré par les seules logiques de rationnement mais aussi par des logiques de marché.

\begin{abstract}
$* * *$
Le marché gazier russe connaît aujourd'hui de profondes évolutions, mais ces dernières n'empruntent pas la voie du modèle dé-intégré de l'U nion européenne. Elles se centrent sur le développement de formes concurrentielles qui permettent à de nouveaux acteurs de contester progressivement la position dominante de Gazprom sur le marché intérieur. L'émergence de la concurrence a été rendue possible grâce aux augmentations progressives des prix du gaz, augmentations qui devraient se poursuivre. Ces changements ne seront pas sans conséquence sur la stratégie d'exportation gazière de la Russie. Un marché intérieur de plus en plus rentable permet d'introduire un certain nombre de flexibilités dans les stratégies d'exportation de Gazprom. Sa nouvelle politique de défense des prix en Europe au détriment de ses parts de marché en est sans doute une première illustration.
\end{abstract}

\title{
RÉFÉRENCES BIBLIOGRAPHIQUES
}

A DACHI Y . (2009), "Subsoil Law Reform in R ussia under the Putin A dministration", Europe-Asia Studies, V ol. 61, $\mathrm{n}^{\circ} 8, \mathrm{pp} .1393-1414$.

AHREND R. \& TOMPSON W. (2004), "Russia's Gas Sector: The Endless Wait for Reform ?", Economics D epartment Working Paper $n^{\circ} 402$, Paris : OECD.

Deacon R. \& Mueller B. (2004), "Political Economy and Natural Resource Use", UCSB Working paper, University of California.

Dubek D., Golub A. \& Strukova E. (2006), "Should Russia increase domestic prices for natural gas?", Energy Policy, Vol. 34, n 13, pp. 1659-1670.

EARLE J. \& ESTRIN S. (1996), "Employee Ownership in Transition", in R. Frydman, C. Gray \& A. Rapaczynski, eds., Corporate Governance in Central Europe and Russia, V ol. 2, Central European University Press.

LoCATELLI C. \& FINON D. (2003), «L'échec de l'introduction d'institutions de marché dans une économie en transition : les limites du consensus de W ashington dans un secteur de rente », Cahiers de recherche LEPII-EPE, $n^{\circ} 2003 / 2$. 
Hedlund S. (2001), "Property without Rights: Dimensions of Russian Privatisation", Europe-Asia Studies, Vol. $53, n^{\circ} 2$, pp. 213-237.

HENDERSON J. (2012), Is a Russian Domestic Gas bubble Emerging ?, Oxford Energy Comment, Oxford Institute for Energy Studies.

Henderson J. (2011), "D omestic Gas Prices in Russia: Towards Export Netback?" ,Working paper NG57, Oxford Institute for Energy Studies.

Henderson J. (2010), Non-G azprom G as Producers in Russia, Oxford Institute for Energy Studies.

KRYUKOV V. \& M OE A. (1996), The new russian corporatism? A case study of Gazprom. Londres: Post-Soviet Business Forum, Royal Institute of International A ffairs.

LOCATELLI C. (2003), "The viability of deregulation in the Russian gas industry", Journal of Energy and Development, vol. 28, n², pp. 221-238.

Nem TSOV B. \& M ILOV V . (2008), Putin and Gazprom; An independant expert report. M oscow

NORTH D. (1991), "Institutions and Credible Commitment", J ournal of Institutional and Theoretical Economics, vol $149, n^{\circ} 1$.

Runov A. (2004), "Demand for Private Property Right in Post-Soviet Russia: Causes and Effects in M anufacturing and Extractive Industries", 8 th Annual Conference of the International Society for New Institutional Economics (ISNIE), 30 September - 30 ctober.

SOROKINE I., ESKIN V. \& WILSON J. (1995). Natural gas industry of Russia: diagnostic review and discussion of policy issues. Prepared for the M inistry of Economy of the Russian Federation, Program on $\mathrm{N}$ atural M onopolies, July.

STERN J . (2005), The Future of Russian gas and Gazprom, Oxford Institute for Energy Studies.

TARR D. (2010), "Export restraints on Russian Natural Gas and Raw timber: What are the Economic Impacts?", CEPE Working paper $n^{\circ} 74$, Zürich.

TARR D. \& THOMSON P. (2003), The Merits of Dual Pricing of Russian Natural Gas, Washington D.C. : World B ank.

TOMPSON W. (2008), "Back to the future? Thoughts on the Political Economy of Expanding State Ownership in Russia", Cahiers Russie, nº, CERI, Paris.

VICTOR N. (2008), "Gazprom: Gas Giant U nder Strain"; PESD Working Paper n 71, Stanford U niversity.

VICTOR N. (2007), "On M easuring the Performance of National Oil Companies", PESD Working Paper $n^{\circ} 64$, Stanford University.

VICTOR N. \& SAYFER I. (2009), «Gazprom: The struggle for power » in VICTOR D, HULTS D., THURBER M. (2009), Oil and governance: State-O wned Enterprises and the World Energy. B usiness \& Economics

WOLF C. (2009), "Does ownership matter? The performance and efficiency of State Oil vs. private oil (19872006)", Energy Policy, Vol. 37, n 7, pp. 2642-2652.

WILLIAM SON O. (1996), The M echanisms of Governance, Oxford U niversity Press, Oxford.

WILLIAM SON O. (1985), The Economic Institutions of Capitalism: Firms, Markets, Relational Contracting, The Free Press, N ew Y ork.

W ood M AcKenzie (2004), Time to Step on the Gas. Will Russia Realise its Potential? M ulti-Client Study 